\title{
GANANCIA GENÉTICA ESPERADA EN MELINA (Gmelina arborea Roxb.) EN CÓRDOBA (COLOMBIA)
}

\section{EXPECTED GENETIC GAIN IN MELINA (Gmelina arborea Roxb.) IN CORDOBA (COLOMBIA)}

\author{
Miguel M. Espitia', Olman Murillo², Carlos Castillo ${ }^{3}$ \\ Recibido para publicación: Enero 22 de 2015 - Aceptado para publicación: Mayo 22 de 2015
}

\begin{abstract}
RESUMEN
En el departamento de Córdoba (Colombia) se tiene proyectado sembrar 100.000 ha de especies forestales para el año 2025. Dentro de las especies recomendadas para esta zona, se encuentra la melina. El objetivo del estudio fue estimar la ganancia genética esperada (GG) en la selección de árboles plus en el Departamento de Córdoba. Se evaluaó el diámetro a la altura del pecho (DAP), altura comercial (ALCO), volumen comercial (VOLCO) y calidad del fuste (CALI). La selección se basó en la evaluación fenotípica del árbol candidato y sus cuatro mejores vecinos en un radio de $20 \mathrm{~m}$. Los árboles seleccionados se clasificaron en lista A cuando superaron en volumen y calidad, con base en el diferencial de selección (S) y en lista B, cuando superaron en volumen o en calidad a todos sus vecinos. La GG se estimó mediante la fórmula: GG= S*h2, donde h2 es la heredabilidad en sentido estrecho promedio reportada. Se construyó un índice de selección (IS), que integró de forma ponderada el VOLCO (60\%) con CALI (40\%). De 57 árboles seleccionados, 35 fueron clasificados como plus A. Al seleccionar y clonar los 35 árboles A, se esperan ganancias genéticas de 4,95\%; $15,47 \% ; 36,65 \%$ y $34,25 \%$, para el diámetro, altura comercial, volumen comercial y calidad del fuste, respectivamente. Los resultados sugieren un progreso genético importante de la melina en el departamento de Córdoba, Colombia, siempre y cuando se amplíe la base genética y se compruebe los resultados mediante ensayos genéticos en varias zonas productoras potenciales.
\end{abstract}

Palabras clave: melina, arboles élite, diferencial de selección, volumen comercial, calidad, índice de selección.

\begin{abstract}
The forest plan in the deparment of Córdoba (Colombia) include to establish 100.000 ha of forest plantations by the year 2025. Melina has been recommended for this region. The objective of this investigation was to estimate the expected genetic gain on melina plus tree. The diameter at breast height, commercial height, trade volume and log quality were assessed. The selection was based on phenotypical evaluation of candidate tree and its four best neighbors within a radius of $20 \mathrm{~m}$. Selected trees were classified in A or B list based on

1'Docente investigador Universidad de Córdoba. I.A. Ph.D. mespitia@sinu.unicordoba.edu.co;Diagonal 50 No. 8 - 105 - Barrio Villa del Río Montería (Córdoba), Colombia. Autor para correspondencia.

${ }^{2}$ Profesor Titular/investigador Instituto Tecnológico de Costa Rica. I.F. Ph.D. olmuga@yahoo.es ; Apartado 1597050 Cartago-Costa Rica ${ }^{3}$ Ingeniero Agrónomo. Universidad de Córdoba. carloscastillo1232@hotmail.com; Calle 15A \#14B-51 (B. Urbina) -Montería (Córdoba), Colombia.
\end{abstract}


the differential selection value (S). Trees were included in A list when its volume and quality were higher than their four best neighbors or in B list when its volume or quality was higher than their neigbors. The GG was estimated by $\mathrm{GG}=\mathrm{S}^{*} \mathrm{~h} 2$, where $\mathrm{h} 2$ is the narrow sense heritability average reported. A Selection Index $(\mathrm{SI})$, that integrated the trade volume and log quality values (60 and $40 \%$ of weight, respectively), was determined. From 57 selected trees, 35 were classified in A plus list. The expected GG from A plus trees will be 4,95\%; $15,47 \% ; 36,65 \%$ y $34,25 \%$ for the diameter at breast height, commercial height, trade volume and log quality, respectively. This results suggest an important genetic progress in the breeding of melina in Córdoba, Colombia.

Key words: words: melina, elite trees, selection differential, commercial volume, quality, selection index.

\section{INTRODUCCIÓN}

Colombia cuenta con 25 millones de ha aproximadamente, con aptitud forestal, de las cuales el departamento de Córdoba posee 897.086 ha (CONIF 2003; MADR 2005; Rincón 2009; CFC 2011). En Córdoba existen 35.000 ha de plantaciones forestales (nativas e introducidas) y en los próximos 25 años se espera plantar 100.000 ha en las principales zonas productoras (Espitia et al. 2014). Entre las especies introducidas plantadas para la producción de madera con fines comerciales, en orden de mayor participación, se encuentran teca (Tectona grandis L.), acacia (Acacia mangium Willd.) y melina (Gmelina arborea Roxb.), con un 25,8\%, 24,9\% y 5\% del área plantada, respectivamente. En ese mismo orden, son las que proveen la mayor cantidad de madera de alta calidad para abastecer la demanda en el mercado internacional (CFC 2011; Espitia et al. 2014).

La melina es considerada una de las especies más promisorias para usaren diferentes procesos industriales y en programas de reforestación, agroforestales y silvopastoriles. Su principal cualidad es su acelerado crecimiento en los primeros seis años, turnos cortos (14 años) y alta producción de biomasa. Alcanza hasta $30 \mathrm{~m}$ de altura y entre 60 a $100 \mathrm{~cm}$ de DAP (Obregón 2006). Su madera es versátil para la elaboración de productos de calidad de mediano a alto valor. Se adapta muy bien en hábitats que varían desde húmedos hasta secos y es una especie oportunista en los bosques húmedos. Sus requerimientos de suelos y climas son intermedios, en comparación con A. mangium y T. grandis (Rojas y Murillo 2004). En Colombia ha tenido excelente adaptación en diferentes zonas productoras de madera, incluida la Región Caribe (CFC 2011; Espitia et al. 2014).

Los principales problemas para la producción forestal en Colombia y en el departamento de Córdoba, específicamente con G. arborea, están relacionados con bajo rendimiento, escasez de semilla (sexual o asexual) como material base para atender la demanda de siembra, dificultad para importar semilla (sexual o asexual) mejorada para siembra, y la ausencia de un programa de mejoramiento genético en la región (Espitia et al. 2010; Espitia et al. 2011; CFC 2011).

El éxito de un programa de mejoramiento genético depende de la calidad e intensidad 
de selección (rigor) de los árboles parentales. Las ganancias esperadas dependen tanto del control genético de las características de interés como de la variabilidad existente en la población (Zobel \& Talbert 1988; Balcorta \& Vargas 2004; Pavlotzky-Blank \& Murillo et al. 2012; Verardi et al. 2013). La heredabilidad en sentido estricto y el diferencial de selección son útiles para predecir la respuesta de la selección en especies forestales (Zobel \& Talbert 1988). El diferencial de selección es importante, porque está altamente correlacionado con la ganancia genética, que es el fin de un programa de mejoramiento genético (Balcorta \& Vargas 2004).

De manera general, se ha utilizado el diferencial de selección fenotípico obtenido durante la selección de los árboles plus, como base para estimar el progreso genético esperado en teca y acacia (Vallejos et al. 2010; Espitia et al. 2010; Espitia et al. 2011). Las estimaciones de ganancia genética esperada le permiten al mejorador forestal conocer su progreso genético potencial y decidir al inicio del programa, cuáles individuos componen la población comercial y cuáles la población de mejoramiento (Vallejos et al. 2010).

Existen diversos reportes de varios países sobre intensidad de selección y ganancias genéticas importantes para varios caracteres de interés del árbol en diferentes especies forestales (Kumar \& Matharoo 2003a; Kumar \& Matharoo 2003b; Botrel et al. 2007; SangUrk et al. 2007; Blada \& Popescu 2008; Oh et al. 2008; Rocha et al. 2009; Verryn et al. 2009; Vallejos et al. 2010; Murillo 2011; Godoy \& Rosado 2011; Pavlotzky-Blank \& Murillo-Gamboa 2012; Mora \& Saavedra 2012; Verardi et al. 2013).
En Colombia se han reportado ganancias genéticas esperada, para caracteres como el DAP, altura total y forma del fuste en varias especies nativas: Alnus jorullensis; Cariniana pyriformis; Cordia alliodora; Genipa americana y Tabebuia rosea (Rodríguez \& Nieto 1999), así como para teca (Espitia et al. 2011) y Acacia mangium (Espitia et al. 2010), sin embargo no se reportan trabajos similares con G. arborea.

El estudio partió de la hipótesis que en las plantaciones donde se seleccionaron los árboles, existía variabilidad genética que podía ser aprovechada por selección y corroborada a través del objetivo principal de este trabajo, el cuál fue estimar la ganancia genética esperada, con base en la selección fenotípica de los mejores 35 árboles plus seleccionados en plantaciones comerciales de melina en los departamentos de Córdoba y Magdalena.

\section{MATERIALES Y MÉTODOS}

El estudio se realizó durante los años 2008 al 2012, en plantaciones comerciales de melina (G. arborea Roxb.) en los departamentos de Córdoba y Magdalena (Colombia), de siete a diez años de edad, las cuales sumaron un total de 200 ha (Tabla 1).

El proceso de selección de los árboles plus se realizó mediante visita y participación de trabajadores de las plantaciones, utilizando la metodología propuesta por Zobel \& Talbert (1984) y adaptada por Vallejos et al. (2010).

La selección de los árboles ocurrió en dos fases, a) preselección de los mejores árboles candidatos de toda la plantación, y b) sanción 
Tabla 1. Localización (municipios y finca) de las plantaciones donde se realizó la selección de árboles plus de Gmelina arborea en el departamento de Córdoba, Colombia.

\begin{tabular}{ccccc}
\hline Municipio & Finca & $\begin{array}{c}\text { Área } \\
\text { reforestada } \\
\text { (ha) }\end{array}$ & \multicolumn{2}{c}{ Coordenadas } \\
Latitud & Longitud \\
\hline Tierralta, Córdoba & 3F Kanguroid & 50 & $08^{\circ} 02^{\prime} 05,5^{\prime \prime}$ & $076^{\circ} 12^{\prime} 01,2^{\prime \prime}$ \\
Zapayán, Magdalena & Hacienda La Gloria & 150 & $93^{\circ} 56^{\prime} 67^{\prime \prime}$ & $161^{\circ} 86^{\prime} 86^{\prime \prime}$ \\
\hline \multicolumn{1}{c}{ Total } & & 200 & & \\
\hline
\end{tabular}

o verificación de la superioridad fenotípica de los candidatos preseleccionados. La primera fase del procedimiento se basa en una revisión exhaustiva de toda el área plantada, buscando los fenotipos sobresalientes en altura total, altura comercial, calidad del fuste y volumen. Por tanto, esta preselección implica una primera estimación de la intensidad de selección "i", que consiste en la relación de los elegidos vs la población total original de individuos de la plantación. La segunda fase de selección, revisa las características del árbol candidato y se compara contra los mejores vecinos, tal y como se explica en detalle más adelante. Los candidatos que logran superar la fase de verificación se constituyen en árboles plus, que conforman entonces la población base de mejoramiento. Su número es utilizado entonces como estimador de la intensidad de selección "i" de la población original que dio origen a la plantación.

Todos los árboles pre-seleccionados y sancionados en cada lote fueron identificados y georeferenciados, para poder colectar su semilla posteriormente. La selección se basó en la evaluación fenotípica del árbol candidato y sus cuatro mejores vecinos en un radio de $20 \mathrm{~m}$, considerando los siguientes caracteres: a) ausencia de gambas, b) rectitud del fuste, c) ausencia de nudos prominentes, d) tamaño y simetría de copa, e) presencia de ramas delgadas con ángulo de inserción de 45 a $90^{\circ}$, f) dominancia en altura, g) sanidad del árbol, h) diámetro a la altura del pecho (DAP), i) altura comercial $\left(\mathrm{h}_{\mathrm{COM}}\right)$ y $\left.\mathrm{j}\right)$ calidad del fuste (CALI); calificando en forma individual las primeros cuatro trozas de 2,5m de largo basado en una escala de " 1 " a " 4 ", donde un valor de " 1 " es la mejor calidad posible y un valor de "4" se asigna para trozas sin valor como madera sólida (Murillo \& Badilla 2004).

El volumen comercial $\left(\mathrm{Vol}_{\mathrm{COM}}\right)$ se estimó utilizando la función que incorpora el DAP y la $\mathrm{h}_{\mathrm{COM}^{\prime}}$ (Murillo \& Badilla 2004) así: $\mathrm{Vol}_{\mathrm{COM}}=\left[(\mathrm{DAP} / 100) 2 * 0,7854 * \mathrm{~h}_{\mathrm{COM}} * 0,65\right]$. La CALI del árbol se estimó con el promedio ponderado de la calidad individual de sus primeros cuatro trozas comerciales. El peso ponderado de la troza en el fuste se basó en su aporte al volumen total de los primeros 10 metros de fuste (Murillo y Badilla 2004).

El valor de calidad del árbol (CALI) se convierte en una variable cuantitativa que registra valores de "1" a "4". Los árboles seleccionados se clasificaron en dos listas A y B. En la lista $A$ se incluyeron los árboles que registraron superioridad tanto en $\mathrm{Vol}_{\text {Сом }}$ como en CALI, con base en el diferencial de selección:

$S=\left[\left(Y_{\text {árbol seleccionado }}-\tilde{Y}_{\text {árboles vecinos }}\right) \tilde{Y}_{\text {árboles vecinos }} * 100\right]$ y en la lista $B$, cuando superaron solamente en $\mathrm{Vol}_{\mathrm{COM}}$ ó en CALI a todos sus mejores vecinos. 


$$
\begin{array}{ll}
\text { Troza } 1=40 \% & (0 \text { a } 2,5 \mathrm{~m}) \\
\text { Troza } 2=30 \% & (2,51 \text { a } 5 \mathrm{~m}) \\
\text { Troza } 3=20 \% & (5,01 \text { a } 7,5 \mathrm{~m}) \\
\text { Troza } 4=10 \% & (7,51 \text { a } 10 \mathrm{~m})
\end{array}
$$

La CALI se transformó de la escala original de "1" a "4" a una escala de 1 a 100 para facilitar su comprensión e interpretación, así: $\mathrm{CALI}_{\text {Invertida }}=100 *\{1-[(\mathrm{CALI}-1) / 3]\}$. Con los valores obtenidos de $\mathrm{S}$, se estimó el diferencial de selección promedio por: i) lote o plantación, ii) todos los árboles seleccionados y iii) árboles seleccionados en la lista A (Zobel \& Talbert 1984; Vallejos et al. 2010; Espitia et al. 2011).

La ganancia genética (GG) se estimó a través de la siguiente ecuación (Zobel \& Talbert 1984; Murillo et al. 2004;; Vallejos et al. 2010):

$\mathrm{GG}=\mathrm{S} * \mathrm{~h} 2$

Donde: "S" es el diferencial de selección y h2 es la heredabilidad en sentido estricto promedio reportada para un grupo amplio de especies tropicales: $h 2_{\text {Diámetro }}=0,20 ; h 2_{\text {Altura }}$ $=0,25 ; \mathrm{h} 2_{\text {volumen }}=0,25 \mathrm{y} \mathrm{h} 2_{\text {Calidad }}=0,35$ (Cornelius 1994).

Con la GG obtenida, se construyó un índice de Selección (IS) que integró de forma ponderada el Vol ${ }_{\text {Сом }}$ con la CALI, así (Murillo et al. 2004; Vallejos et al., 2010):

IS $=\left[\left(0,6 *\left(\mathrm{Vol}_{\mathrm{COM}}-\right.\right.\right.$ Promedio del volumen comercial $) / \mathrm{ds})+\left(0,4^{*}(\mathrm{CALI}-\right.$ Promedio de la calidad)/ds)]
Donde:

ds = desviación estándar de cada carácter.

$\mathrm{Vol}_{\mathrm{COM}}=$ Promedio del volumen comercial del conjunto de los árboles plus.

$\mathrm{CALI}=$ Promedio de la calidad del conjunto de los árboles plus

Promedio $=$ se refiere al promedio de todos los árboles involucrados en la evaluación fenotípica de los árboles plus (vecinos + árboles plus).

Los coeficientes de 0,6 (para $\mathrm{Vol}_{\mathrm{COM}}$ ) y 0,4 (para CALI), son el peso económico asignado a la variable.

Con base en el "IS" se obtuvieron los mejores árboles plus, tanto en volumen comercial como en calidad, los cuales pasan a constituir la subpoblación comercial (plus A) inicial. El resto de los árboles (plus B) forman parte de la población de mejoramiento e investigación. Todos los cálculos en este estudio se realizaron en la hoja electrónica de Excel.

\section{RESULTADOS Y DISCUSIÓN}

\section{Árboles plus seleccionados e intensidad de selección.}

En la tabla 2 se puede observar que el proceso de selección realizado permitió identificar un total de 57 árboles plus por sus características fenotípicas sobresalientes $(A+B)$. El número de árboles seleccionados por finca osciló entre 21 y 36. Esta variación se debe fundamentalmente a la variación en tamaño de las plantaciones, 
las cuales oscilaron entre 50 y 150 ha (Tabla 1). Probablemente, otro factor que afectó el número de árboles seleccionados por finca, fue la variabilidad y el origen genético de la semilla sexual (material procedente de áreas productoras de semilla de libre polinización, utilizado para la siembra de las plantaciones objeto de estudio). Esto fue posible detectar en la visita de selección y eliminación de árboles en las plantaciones, hasta el punto que en algunos lotes muestreados dentro de una misma finca, no se incluyó ningún árbol superior.

Con base en el proceso de selección descrito se obtuvo una intensidad de selección por finca que osciló entre 1 por cada 2600 (3F Kanguroid) a 1 por cada 4.600 árboles (Hacienda La Gloria). Esto originó una intensidad de selección promedio de 1 árbol seleccionado por cada 4.100 árboles evaluados (Tabla 2), que equivale aproximadamente a 1 árbol por cada 4,0 ha, lo cual demuestra parte del rigor y exigencia en el proceso de selección de los mejores árboles en las plantaciones revisadas en Córdoba y Magdalena.

De los 57 árboles seleccionados, el 61\%, aproximadamente (35 árboles), fueron clasificados como plus $A$, ya que superaron a sus mejores vecinos en los criterios de volumen y calidad. Mientras que el 39\% restante de los árboles seleccionados (22), sólo superaron a sus árboles vecinos en volumen o en calidad (integrantes de la lista de árboles Plus B). La intensidad de selección para los 35 árboles plus A, fue de 1 cada 6.350 árboles. Esta intensidad de selección promedio para este grupo de árboles, resultó ser más exigente que la reportada por Balcorta \& Vargas (2004), quienes reportan la selección de 1 árbol plus por cada 1.111 individuos en G. arborea; pero inferior a la mencionada por Murillo \& Badilla (2009), Espitia et al. (2010 y 2011) y Pavlotzky-Blank \& Murillo-Gamboa (2012), quienes reportan 1 árbol seleccionado por cada 15.000 a 20.000 individuos en G. arborea, 1 árbol plus por cada 10.622 a 30.538 individuos en A. mangium y 1 árbol por cada 20.000 a 36.193 individuos, en T. grandis, respectivamente. Igualmente, las intensidades de selección fueron superiores a las recomendadas por Zobel \& Talbert (1984), quienes sugieren intensidades de selección de 1: < 20 para rodales semilleros y 1: > 1000 para huertos semilleros. Los valores de intensidad de selección obtenidos en este estudio, se consideran suficientes para iniciar un programa de mejoramiento genético, ya que además de permitir identificar un número importante de árboles superiores, también

Tabla 2. Árboles seleccionados e intensidad de selección por lote en las poblaciones estudiadas de G. arborea en el departamento de Córdoba y Magdalena (Colombia).

\begin{tabular}{|c|c|c|c|}
\hline Municipio & Finca & $\begin{array}{c}\text { Árboles plus } \\
\text { seleccionados (\#) }\end{array}$ & $\begin{array}{l}\text { Intensidad de Selección } \\
\text { (árboles) }\end{array}$ \\
\hline Tierralta, Córdoba & 3F Kanguroid & 21 & 1 cada 2.600 (2,5 ha) \\
\hline Zapayán, Magdalena & Hacienda La Gloria & 36 & 1 cada $4.600(4,5$ ha $)$ \\
\hline Global $(A+B)$ & & 57 & 1 cada 4.100 (4 ha) \\
\hline Plus A & & 35 & 1 cada 6.350 (5,7 ha) \\
\hline
\end{tabular}


hace posible racionalizar y hacer eficiente el proceso de mejoramiento, sin generar problemas importantes de endogamia en la progenie resultante.

\section{Diferencial de selección, ganancia genética esperada e índice de selección.}

En la tabla 3 se observa que el diferencial de selección promedio (\%) varió con el tipo de lotes (departamento) y los cuatro caracteres evaluados. Aun cuando el número de árboles seleccionado en cada departamento fue diferente, en Córdoba se obtuvieron los mayores diferenciales de selección para altura $\left(\mathrm{h}_{\mathrm{COM}}\right)$ y volumen comercial $\left(\mathrm{Vol}_{\mathrm{COM}}\right)$, con valores de 76,11 y 130,7\%, respectivamente. Mientras que en Magdalena se obtuvieron las mayores estimaciones de diferencial de selección para diámetro a la altura del pecho (DAP) y en calidad del fuste (Calidad), con valores de 12,41 y 78,06\%, respectivamente. Entre los cuatro caracteres, el mayor diferencial de selección fue registrado en el $\mathrm{Vol}_{\mathrm{COM}^{\prime}}$ seguido por la Calidad, $\mathrm{h}_{\text {Сом }}$ y DAP del fuste, con valores promedios ponderados de 81,13; 70,$95 ; 39,60$ y $11,82 \%$, respectivamente.
Estos resultados son similares en tendencia y magnitud a los estimados en Acacia mangium y Tectona grandis por Espitia et al. (2010 y 2011) y Pavlotzky-Blank \& Murillo-Gamboa 2012, pero superiores a los reportados en melina por Kumar \& Matharoo (2003a) y Balcorta \& Vargas (2004), con valores de diferencial de selección de $40 \%$ para altura total y $40 \%$ para volumen comercial respectivamente.

Sin embargo, se debe tener presente como lo señalan Vallejos et al. (2010), que estos valores de diferencial de selección son por lo general más bajos de lo real, debido a que cada árbol plus fue evaluado contra sus mejores cuatro vecinos. Se puede inferir que estos vecinos son competidores muy fuertes e igualmente, constituyen parte de los mejores individuos de la población base ordinaria (sin mejoramiento) que usualmente se obtiene de los viveros comerciales. Por lo tanto, el verdadero diferencial de selección que se presenta en este tipo de programas, usualmente es superior y supera notablemente a la población base.

Tabla 3. Diferencial de selección promedio (S) de los árboles plus de G. arborea seleccionados en el departamento de Córdoba y Magdalena (Colombia).

\begin{tabular}{|c|c|c|c|c|c|c|}
\hline Departamento & $\begin{array}{l}\text { Árboles plus } \\
\qquad(\#)\end{array}$ & $\begin{array}{c}\text { S } \\
\text { DAP* } \\
(\%)\end{array}$ & $\begin{array}{c}\mathbf{S} \\
\mathbf{h}_{\mathrm{COM}} \\
(\%)\end{array}$ & $\begin{array}{c}\mathbf{S} \\
\text { Vol }_{\text {COM }} \\
(\%)\end{array}$ & $\begin{array}{c}\text { S } \\
\underset{(\%)}{\text { Calidad }}\end{array}$ & $\begin{array}{l}\text { Indice de } \\
\text { Selección } \\
\text { (IS) } \\
(\%)\end{array}$ \\
\hline $\begin{array}{l}\text { Tierralta, 3F Kanguroid (Córdoba) } \\
\text { Zapayán, Magdalena }\end{array}$ & $\begin{array}{l}21 \\
36\end{array}$ & $\begin{array}{l}10,81 \\
12,41\end{array}$ & $\begin{array}{l}76,11 \\
18,30\end{array}$ & $\begin{array}{l}130,7 \\
52,21\end{array}$ & $\begin{array}{l}76,11 \\
18,30\end{array}$ & $\begin{array}{c}274,09 \\
72,40\end{array}$ \\
\hline $\begin{array}{l}\text { Promedio ponderado de todos los } \\
\text { árboles plus en las dos regiones } \\
(\mathrm{A}+\mathrm{B})\end{array}$ & 57 & 11,82 & 39,60 & 81,13 & 70,95 & 146,71 \\
\hline $\begin{array}{l}\text { Promedio ponderado de los Plus } \\
\text { A en las dos regiones }\end{array}$ & 35 & 19,81 & 51,56 & 122,6 & 76,11 & 197,23 \\
\hline
\end{tabular}

*Diámetro a la altura del pecho (DAP), Altura comercial $\left(\mathrm{h}_{\mathrm{COM}_{\mathrm{M}}}\right)$; Volumen hasta altura comercial $\left(\mathrm{Vol}_{\mathrm{COM}}\right)$; Calidad del fuste en una escala de 0 a 100, donde 100 es excelente; Índice de Selección (integra Volumen Comercial*0,6 con la calidad*0,4). 
La misma tendencia en el diferencial de selección, se puede observar en los cuatro caracteres evaluados, cuando sólo se consideran los 35 árboles plus A. Los valores más altos se registraron en: $\operatorname{Vol}_{\mathrm{COM}}(122,16 \%), \mathrm{CALI}(76,11 \%)$, $\mathrm{h}_{\text {COM }}(51,56 \%)$, y DAP $(19,81 \%)$. Estos resultados reflejan la superioridad genética potencial de los 35 árboles plus $\mathrm{A}$, frente a los 57 árboles totales seleccionados $(A+B)$, especialmente en $\mathrm{Vol}_{\mathrm{CO}}(41,03$ puntos más en porcentaje) y $\mathrm{h}_{\text {COM }}(11,96$ puntos más en porcentaje). Los valores de diferencial de selección registrados en este estudio para los plus $A$, son similares en tendencia y magnitud a los encontrados en A. mangium y T. grandis por Espitia et al. (2010 y 2011), y mayores a los reportados por: Vallejos et al. (2010) en teca en el Pacífico central de Costa Rica de $22,88 \%$ en volumen y $21,83 \%$ en calidad; igualmente a los encontrados por Murillo \& Badilla (2003) en teca, con diferencial de selección para el volumen comercial de $24 \%$ superior en las mejores familias y de $39 \%$ más que los testigos. Así mismo, a los valores relacionados por Balcorta \& Vargas (2004), quienes han registrado diferenciales de selección de un $40 \%$ para altura y $40 \%$ para volumen comercial respectivamente, con respecto a la población original.

Es importante destacar que los resultados del proceso de selección indican que los mayores índices de selección (IS) se presentaron en las plantaciones de 3F Kanguroid - Córdoba (IS= 274,09\%), superando en 201,69 puntos en porcentaje a las plantaciones de Zapayán - Magdalena (Tabla 3). Estos resultados evidencian que en las plantaciones de Córdoba se encuentra la mayor cantidad de árboles seleccionados, los cuales reúnen las mejores características en crecimiento, volumen y calidad del fuste. Además, se presenta una superioridad en el índice de selección de los 35 árboles plus $\mathrm{A}(\mathrm{IS}=197,23 \%)$ sobre los 57 árboles seleccionados (IS= $146,71 \%)$, esto se explica porque todos los individuos plus A, superan en volumen y calidad a todos sus mejores vecinos o testigos. Estos resultados son superiores a los encontrados en Córdoba por Espitia et al. (2010 y 2011) en procesos de selección de A. mangium y T. grandis, con valores fenotípicos promedio de índices de selección para los árboles plus A de 126,08\% y $37,90 \%$, respectivamente.

Al comparar los valores promedios para $h_{\text {COM }}$ y Calidad del fuste de los 35 árboles plus A, frente al grupo total de 228 mejores vecinos utilizados como testigos (Tabla 4), se puede estimar un incremento fenotípico de los árboles plus $\mathrm{A}$ de $51,56 \%$ y $76,11 \%$, en altura comercial $\left(\mathrm{h}_{\mathrm{COM}}\right)$ y calidad del fuste $(\mathrm{CALI})$, respectivamente.

En la tabla 5 se presentan los estimados de ganancia genética esperada (GG) e índice de selección (IS) en los cuatro caracteres estudiados, si se utilizan los 57 árboles plus seleccionados $(A+B)$ o se utilizan los 35 árboles plus $\mathrm{A}$, a partir de su semilla sexual o directamente como clon. Entre los cuatro caracteres analizados en el proceso de selección los mayores niveles de ganancia genética esperada se estimaron en los caracteres calidad del fuste y volumen comercial $\left(\mathrm{Vol}_{\mathrm{COM}}\right)$. Tanto cuando se emplea la semilla sexual o se toma la decisión de clonar los árboles seleccionados. La ganancia genética para calidad de fuste osciló entre 28,38\% (árboles A+B, semilla) y 34,25\% (árboles A, clon). Mientras que para el $\mathrm{Vol}_{\mathrm{COM}}$ las ganancias variaron desde 20,28\% (árboles $A+B$, semilla) 
Tabla 4. Árboles seleccionados e intensidad de selección por lote en las poblaciones estudiadas de G. arborea en el departamento de Córdoba y Magdalena (Colombia).

\begin{tabular}{lccccc}
\hline \multicolumn{1}{c}{ Grupo } & $\begin{array}{c}\text { Árboles plus } \\
(\#)\end{array}$ & $\begin{array}{c}\mathbf{h}_{\text {Com }} \\
(\mathbf{m})\end{array}$ & $\begin{array}{c}\text { Diferencial } \\
\mathbf{h}_{\text {com }} \\
(\mathbf{m})\end{array}$ & Calidad & $\begin{array}{c}\text { Diferencial } \\
\text { Calidad(\%) }\end{array}$ \\
\hline Plus A & 35 & 15,71 & 51,56 & 82,19 & 76,11 \\
Plus (A+B) & 57 & 14,86 & 39,60 & 81,23 & 70,95 \\
Testigos (mejores vecinos) & 228 & 11,86 & & 47,42 & \\
\hline
\end{tabular}

*Altura comercial $\left(\mathrm{h}_{\mathrm{COM}}\right)$ y Calidad del fuste de 0 a 100 , donde 100 es excelente.

Tabla 5. Ganancia genética (GG) esperada por categoría de árboles plus de G.arborea seleccionados en el departamento de Córdoba y Magdalena (Colombia).

\begin{tabular}{ccccc}
\hline \multirow{2}{*}{ Caracteres } & \multicolumn{4}{c}{ GG (\%) } \\
\cline { 2 - 5 } & (A + B, semilla) & (A, semilla) & (A + B, clon) & (A, clon) \\
\hline DAP & 2,36 & 3,96 & 2,96 & 4,95 \\
hCOM & 9,90 & 12,89 & 11,88 & 15,47 \\
VolCOM & 20,28 & 30,54 & 24,34 & 36,65 \\
Calidad & 28,38 & 30,44 & 31,93 & 34,25 \\
\hline
\end{tabular}

Indice de

Selección (IS)

* Diámetro a la altura del pecho (DAP), Altura comercial $\left(\mathrm{h}_{\mathrm{com}}\right)$, Volumen comercial $\left(\mathrm{Vol}_{\mathrm{com}}\right)$, Calidad del fuste en una escala de 0 a 100 e Índice de Selección (integra Volumen Comercial*0,6 con la calidad*0,4).

hasta $36,65 \%$ (árboles $\mathrm{A}$, clon), con respecto a los mejores árboles vecinos, considerados como testigos o población base (Tabla 5). Los resultados obtenidos en este estudio son similares o mayores a los reportados por diferentes autores, como Espitia et al. (2010) y Pavlotzky-Blank \& Murillo-Gamboa 2012, quienes en $\mathrm{A}$. mangium reportan ganancias genéticas esperadas en promedio de árboles plus A de $22,24 \%$ y $48,57 \%$ en altura y volumen comercial, respectivamente; Espitia et al. (2011) en selección de T. grandis, detectaron GG promedio en árboles plus $\mathrm{A}$ de $41,71 \%$ y $9,59 \%$, para volumen y calidad del fuste, respectivamente. Al respecto, Vallejos et al. (2010), relacionan ganancias genéticas esperadas en general entre 20 25\% en volumen, y Mesén (2001) estimó ganancias genéticas en melina de $17 \%$ en altura y $43 \%$ en DAP. Por su parte, Cornelius \& Hernández (1994), en la misma especie, reportaron ganancias genéticas de hasta $12 \%$ en rectitud del fuste, y Kumar \& Matharoo (2003a), en melina a nivel clonal, encontraron para altura, diámetro basal y diámetro a la altura del pecho, ganancias de un 18\%, 25\% y 30\%. Finalmente, Rojas \& Arias (2004), en Pinus caribaea var. hondurensis Barr., en la zona del Pacífico sur de Costa Rica, obtuvieron ganancias del $23 \%$ en volumen. Los resultados de este estudio evidencian el riguroso proceso de selección realizado en las plantaciones evaluadas y permiten vaticinar un importante progreso genético en los ensayos de evaluación de progenies y/o clones. 
Se observa en la tabla 5, que se pueden lograr mayores ganancias genéticas en el programa de mejoramiento empleando solamente los 35 árboles plus $\mathrm{A}$, en vez de utilizar todos los 57 árboles seleccionados $(\mathrm{A}+\mathrm{B})$, tanto utilizando su semilla o clonándolos.

Si en el programa de mejoramiento genético de melina se utilizara la semilla sexual de los mejores 35 árboles plus A (A, semilla), se obtendrían ganancias genéticas $(78,89 \%$ versus 58,68\%) mayores al uso de la semilla de los 57 árboles seleccionados (A+B, semilla). En $\mathrm{Vol}_{\mathrm{COM}}$ se lograría un progreso genético de $10,26 \%$ adicional y de $2,06 \%$ adicional en Calidad. Espitia et al. (2010 y 2011) en los procesos de selección de A. mangium y T. grandis, reportan para Córdoba (Colombia), resultados superiores en $\mathrm{Vol}_{\mathrm{COM}}$ con valores de $19,04 \%$ y $12,5 \%$, respectivamente. Mientras que los resultados para calidad del fuste en las dos especies, han sido similares con valores de alrededor del 2,1\%.

En general, para los cuatro caracteres de interés incluido en el estudio, el progreso genético esperado es mayor al clonar los 35 árboles plus $A(A$, clon) que al utilizar su semilla (A, semilla). Los valores de superioridad adicional oscilan entre un $0,99 \%$ (DAP) y un 6,11\% ( $\left.\mathrm{Vol}_{\mathrm{COM}}\right)$ (Tabla 5). De acuerdo con Ipinza (1998), Murillo \& Badilla (2009), Godoy \& Rosado 2011; Pavlotzky-Blank \& Murillo-Gamboa 2012; Mora \& Saavedra 2012 y Verardi et al. 2013, esta diferencia en ganancia genética a favor de la clonación de los árboles plus A, se explica porque en la clonación se captura el $100 \%$ de la información genética (efectos genéticos aditivos y no aditivos) de los árboles plus A; mientras que cuando se utiliza la semilla sexual originada de polinización abierta, sólo se captura el $50 \%$ de la información genética de los árboles plus A seleccionados (madre), ya que no se conoce al progenitor masculino.

La tendencia y superioridad de los valores de ganancia genética esperada determinada en este estudio, son consistentes con los índices de selección (IS) obtenidos, los cuales oscilaron entre 56,68\% (A+B semilla) y $88,75 \%$ (A, clon) para las cuatro estrategias de mejoramiento posibles (Tabla 5). Esto indica que los árboles plus $A$, cuando son clonados (A, clon) generan la mayor ganancia genética esperada en volumen y calidad (IS $=88,75 \%$ ), comparado con el uso de la semilla de tales árboles (A, semilla: IS = 78,89\%) para obtener nuevas progenies.

Sí consideramos que con incrementos del $4 \%$ por conceptos de ganancias genéticas en volumen se cubren los costos de un programa de mejoramiento genético forestal (Ipinza, 1998), los resultados obtenidos en este estudio permiten deducir alta rentabilidad económica en el programa que se adelanta en Córdoba. Ello en razón a que las ganancias genéticas esperadas en $\mathrm{Vol}_{\mathrm{COM}^{\prime}}$ han presentado valores del $30,54 \%$ y $36,65 \%$, cuando se emplea la semilla sexual ( $A$, semilla) o se clonan los árboles $\mathrm{A}(\mathrm{A}, \mathrm{clon})$, respectivamente.

De acuerdo con Murillo \& Badilla (2009), Godoy \& Rosado 2011; Pavlotzky-Blank \& Murillo-Gamboa 2012; Mora \& Saavedra 2012 y Verardi et al. 2013, sí se utiliza la estrategia de clonación de los árboles plus A, en aproximadamente 1 a 2 años se puede iniciar con el establecimiento de plantaciones clonales comerciales con material de alto 
rendimiento. Mientras que sí se utiliza la estrategia de semilla sexual (familias de medios hermanos), en aproximadamente 6 a 8 años se tiene semilla mejorada para abastecer la demanda de plantaciones.

A lo anterior, se le debe adicionar que la melina es una especie que se propaga vegetativamente y rebrota fácilmente. Los árboles plus seleccionados pueden ser incluso talados para iniciar su propagación vegetativa y los nuevos rebrotes pueden iniciar su propagación a partir de 6 a 8 semanas después de cortado el árbol. Estos niveles de progreso genético esperado, permiten adicionalmente, vaticinar una reducción de aproximadamente 1 a 2 años en el tiempo para alcanzar dimensiones de cosecha final, si y solo si, se siguen los principios del manejo oportuno de la plantación (Murillo \& Badilla 2009). Lo cual generará un alto impacto y estímulo a la reforestación con melina en el departamento de Córdoba y en Colombia.

Los 35 árboles plus A, por sus características superiores, pueden ser utilizados como progenitores para siembras comerciales, utilizando su semilla sexual (aprovechamiento sólo de la varianza genética aditiva) o clonándolos directamente (aprovechamiento de toda la varianza genética) para lograr capturar la mayor ganancia genética potencial (Ipinza 1998; Mesén 2001; Murillo \& Badilla 2009; Vallejos et al. 2010; Godoy \& Rosado 2011; Pavlotzky-Blank \& Murillo-Gamboa 2012; Mora \& Saavedra 2012; Verardi et al. 2013). Sin embargo, como se observa en la tabla 5, la mayor ganancia genética esperada en volumen comercial y calidad del fuste, se obtiene con la clonación de los
35 árboles plus A, con valores de 36,65\% y $34,25 \%$, respectivamente. Los árboles plus B (22 restantes), no se incorporarán a la población comercial, dado que representan una condición de superioridad solamente en uno de los dos caracteres volumen ó calidad. Por lo tanto, formarán parte de la población de mejoramiento (investigación y desarrollo) y se mantendrán a la espera de su evaluación genética y su utilización en los cruzamientos controlados en el programa de mejoramiento de melina en Córdoba, como lo señalan Murillo \& Badilla (2009) y Vallejos et al. (2010).

Los resultados encontrados le permiten igualmente al mejorador forestal, de acuerdo a sus recursos económicos y de suelos, definir sí incluye en sus ensayos de progenies y de clones todos los 57 árboles seleccionados $(A+B)$ o evalúa en tales pruebas solamente los 35 árboles plus A. Una prueba de progenie o clonal de los 35 árboles, replicada en al menos tres ambientes diferentes, es aceptable en este tipo de ensayos y permitiría, después del raleo genético, contar con sendos huertos semilleros de primera generación, que ofrecerían semilla de excelente calidad genética para nuevas siembras comerciales. De igual forma se contaría con material vegetal para clonar los mejores árboles de las mejores familias, con adaptación específica en cada ambiente. Esta estrategia haría posible que los árboles definitivos se crucen entre sí, forzando su recombinación genética, la acumulación de alelos favorables y por consiguiente la producción de semilla de calidad genética superior, para continuar con el proceso de mejoramiento (Ipinza 1998; Mesén 2001; Godoy \& Rosado 2011; Pavlotzky-Blank \& Murillo-Gamboa 2012 y Mora \& Saavedra 2012). 
Como es de esperarse, es posible que se encuentren buenos genotipos en otras empresas, organizaciones o países. Por lo tanto, este grupo de árboles plus A que se han seleccionado y dado inicio a un programa de mejoramiento genético de melina en Córdoba, como lo señalan Murillo \& Badilla (2009), permiten igualmente el desarrollo de modelos cooperativos o de alianza e intercambio de material genético con otras empresas $u$ organizaciones. Esta estrategia junto con la introducción de germoplasma de nuevas procedencias fuera del país, hace posible reducir costos, aumentar variabilidad genética y reducir tasas de consanguinidad en la población de mejoramiento, lo que permitirían seguir obteniendo ganancias genéticas durante varias generaciones (Pavlotzky-Blank \& Murillo-Gamboa 2012).

Los resultados obtenidos sugieren un progreso genético significativo en el mejoramiento de G. arborea en Córdoba. De acuerdo con Zobel \& Talbert (1984) y Xavier et al. (2009), este avance integrado con el proceso de silvicultura clonal con los mejores árboles élites, pueden constituir el complemento ideal de un programa de mejoramiento genético para esta especie. Adicionalmente, hacen prever un aporte importante a la productividad, competitividad y sostenibilidad de la producción forestal en esta zona del caribe colombiano, de acuerdo a las exigencias de calidad del mercado internacional al cual se pretende llegar. No obstante es necesario corroborar este progreso genético, mediante ensayos genéticos apropiados en varias zonas productoras de Córdoba.

A pesar de las importantes ganancias genéticas esperadas que se han estimado, vale la pena señalar que los 57 árboles plus localizados en el Departamento de Córdoba y Magdalena, constituyen todavía una base genética relativamente pequeña, para sustentar un programa de mejoramiento genético a largo plazo con esta especie. Por lo anterior, es imprescindible realizar esfuerzos para introducir nuevas procedencias y realizar intercambio de germoplasma, con el fin de ampliar la base genética de este programa.

\section{CONCLUSIONES}

De los 57 árboles seleccionados, 35 (61\%) fueron clasificados como plus A y los 22 (39\%) restantes como plus $\mathrm{B}$.

Al seleccionar y clonar los 35 mejores árboles plus A con base en el IS, se espera obtener ganancias genéticas de 4,95\%; 15,47\%; 36,65 y $34,25 \%$, para los caracteres DAP, $h_{\mathrm{COM}^{\prime}}$ $\mathrm{Vol}_{\mathrm{COM}}$ y CALI, respectivamente.

Los resultados obtenidos sugieren un progreso genético notable en el mejoramiento de Gmelina arborea en el departamento de Córdoba, Colombia.

Es necesario comprobar este gran potencial de mejoramiento genético, mediante ensayos genéticos en varias zonas productoras de Córdoba.

\section{REFERENCIAS}

Balcorta, H. y Vargas, J. 2004. Variación fenotípica y selección de árboles en una plantación de melina (Gmelina arborea Linn., Roxb.) de tres años de edad. Revista 
Chapingo, Serie ciencias forestales y del ambiente. 10(1):13-19

Blada, I. y Popescu, F. 2008. Diallel crossing in Pinus cembra: IV. age trends in genetic parameters and genetic gain for growth and branching traits Ann. For. Res. 51:89-112.

Botrel, M., Moreira da Silva, J., Trugilho, P., Da Silva, S. and Bruno Ricardo, B. 2007. Ganho genético em propiedades físicas e mecânicas de clones de eucalipto. Science Forestry, Piracicaba. 76(1):13-19.

CFC-Cadena Forestal de Córdoba. 2011. Acuerdo regional de competitividad: Cadena forestal madera, muebles y productos de madera del departamento de Córdoba 2011-2030 (Texto y matriz del acuerdo), 55p.

Conif. 2003. Corporación Nacional de Investigación y Fomento Forestal. Cadena Forestal Productiva de Córdoba. Folleto Núcleos Forestales. http://www.conif.org. co/docs/cadena_folleto_interior.pdf [0410-2009].

Cornelius, J. 1994. The effectiveness of plus-tree selection for yield. Forest Ecology and Management. 67:23-34.

Cornelius, J. y Hernández, M. 1994. Variación genética en crecimiento y rectitud del fuste en Gmelina arborea en Costa Rica. Boletín Mejoramiento Genético y Semillas Forestales.10:9-12.

Espitia, M., Araméndiz, H., Montiel, J. 2014. Parámetros de germinación en camára germinativa e invernadero en cinco especies forestales nativas en Córdoba.
Ponencia. Memorias XLIV Congreso Anual de COMALFI (Sociedad Colombiana de Control de Malezas y Fisiología Vegetal). Centro de Convenciones de Córdoba Montería ,Septiembre de 2014, 91 p.

Espitia, M., Murillo, O. y Castillo, C. 2011. Ganancia genética esperada en la selección de teca (Tectona grandis L.) en Córdoba (Colombia). Revista Colombia Forestal 14(1):95-106.

Espitia, M., Murillo, O., Castillo, C.,Araméndiz, H. y Paternina, N. 2010. Ganancia genética esperada en la selección de acacia (Acacia mangium WILLD) en Córdoba (Colombia). Revista U.D.C.A Actualidad \& Divulgación Científica 13(2): 99-107.

Godoy, T. y Rosado, S. da S. 2011. Estimates of genetic gains for growth traits in young plants of Eucalyptus urophylla S. T. Blake. CERNE. 17(2):189-193.

Ipinza, R. 1998. Mejoramiento genético forestal. Serie Técnica No42, Programa CONIF-Ministerio de Agricultura sobre investigaciones en semillas de especies forestales nativas. INSEFOR. Santa fe de Bogotá, Colombia, 162p.

Kumar, A. y Matharoo, AK. 2003. Genetic improvement of Gmelina arborea in India. In: Recent Advances with Gmelina arborea (eds. W. S. Dvorak, G. R. Hodge, W. C. Woodbridge and J. L. Romero). CDROM. CAMCORE, North Carolina State University. Raleigh, NC US. CAMCORE, $221 \mathrm{p}$ 
Kumar, A. y Matharoo, AK. 2003. Growth performance and variability in different clones of Gmelina arborea in India. In Recent Advances with Gmelina arborea (eds. W. S. Dvorak, G. R. Hodge, W. C. Woodbridge and J. L. Romero). CDROM. CAMCORE, North Carolina State University. Raleigh, NC. USA.

MADR, 2005. Ministerio de Agricultura y Desarrollo Rural. 2005. Características y estructura del sector forestal-maderamuebles en Colombia, una mirada global de su estructura y dinámica 1991-2005. Documento de trabajo no. 95. Observatorio Agrocadenas Colombia, 63p.

Mesén, F. 2001. Introducción al mejoramiento genético forestal. En Identificación, selección y manejo de fuentes semilleras. Serie Técnica / No. 32. Convenio CONIF, INSEFOR y MADR Bogotá, septiembre de 2001, 118p.

Mora, F. y Saavedra, J. 2012. Combining genetic gain and diversity under an individual selection method in a selected provenance of Eucalyptus cladocalyx. Cienc. Inv. Agr 39(1):177-184.

Murillo, O. y Badilla, Y. 2004. Evaluación de la calidad y estimación del valor en pie de la plantación forestal. Escuela de Ingeniería Forestal, ITCR. Cartago, Costa Rica, 50 p.

Murillo, O. y Badilla, Y. 2009. Reproducción clonal de árboles. Taller de Publicaciones. Instituto Tecnológico de Costa Rica, ITCR. Cartago, Costa Rica, 45 p.
Murillo, O. 2011. Estrategia de mejoramiento genético forestal para la Cooperativa GENFORES (Costa Rica). Conferencia magistral. En: XII Congreso Nacional de Fitomejoramiento y Producción de Cultivos", Montería, junio de 2011, 57p.

Murillo, O. y Badilla, Y. 2003. Potencial de mejoramiento genético de la Teca en Costa Rica. En Simposio sobre la teca, CD-ROM, Universidad Nacional, Heredia, Costa Rica, noviembre del 2003.

Murillo, O., Obando, G., Badilla, Y. y Araya, E. 2004. GENFORES, a Costa Rican tree improvement and gene conservation cooperative. En: IUFRO Meeting. Forest Genetics and Genomics, CD-ROM, November. Charleston, South Carolina, USA.

Murillo, O., Espitia, M. y Castillo, C. 2012. Fuentes semilleras para la producción forestal. Universidad de Córdoba. Ed. Damar S.A.S Bogotá, 176p.

Obregón, C. 2006. Gmelina arbórea:Versatilidad, renovación y productividad sostenible para el futuro. Revista El Mueble y la Madera. (50):14-20.

Oh, C.Y., Han, S., Mm, C., Kang, K. and Lee, B. 2008. Genetic gain and diversity in a clonal seed orchard of Pinus Koraiensis under various thinning intensities. Korean Journal of Breeding Science. 40(3): 263-268.

Pavlotzky-Blank, B. y Murillo-Gamboa, O. 2012. Ganancia genética esperada en Acacia mangium en Los Chiles, Zona Norte de Costa Rica. Agron. Mesoam. 23(1): 93-106. 
Rincón, M. 2009. El sector forestal en Córdoba: Cadena productiva forestal madera $y$ muebles departamento de Córdoba. Informe Cadena Forestal de Córdoba, Febrero de 2009 (Centro de Investigaciones Turipaná - Corpoica), 37p.

Rocha, R., Vieira, A., Bentes, M.D. y Brum, L.M. 2009. Avaliação genética de procedências de bandarra (Schizolobium amazonicum) utilizando REML/BLUP (Máxima verossimilhança restrita/Melhor predição linear não viciada. Sci. For., Piracicaba 37(84): 351-358

Rodríguez, J. y Nieto, V. 1999. Investigación en semillas forestales nativas. Serie Técnica No. 43. ISSN 0121-0300. Programa de Investigación en Semillas Forestales Nativas - INSEFOR. Convenio CONIF - Ministerio de Agricultura, 89p

Rojas F. y Arias D. 2004. Manual para productores de Melina (Gmelina arborea) en Costa Rica. Cartago, 86p.

Rojas, F. y Murillo, O. 2004. Botánica y Ecología. En: Rojas, F., Arias, D., Moya, R., Meza, A., Olman, M. y Arguedas, M. (Ed). Manual para productores de melina (Gmelina arbórea) en Costa Rica, Cartago, pp. 3-21.

SangUrk, H.; KyuSuk, K.; Byoung Hwan, C. y ChangSoo, K. 2007. Realized genetic gains and heritabilities for height, $\mathrm{DBH}$ and volume growth in open-pollinated progenies of Pinus thunbergii. Korean Journal of Breeding Science 2007. 39(1): 15-19.
Vallejos, J., Badilla, Y., Picado, F. y Murillo, O. 2010. Metodología para la selección e incorporación de árboles plus en programas de mejoramiento genético forestal. Agronomía Costarricense. 33(1): 105-119.

Verardi, C.K., Scaloppi-Junior, E.J., Peres-Silva, G.A., Lima-Gouvêa, L.R. and de SouzaGonçalves, P. 2013. Genetic parameters and estimated genetic gains in young rubber tree progenies. Pesq. agropec. bras. 48(4):411-416.

Verryn, S.D., Snedden,C.L., Eatwell, K.A. 2009. A comparison of deterministically predicted genetic gains with those realized in a South African Eucalyptus grandis breeding program. Journal of Forest Science, Menlo Park. 71(2):141-146.

Xavier, A., Wendling, I. y da Silva, R. 2009. Silvicultura Clonal. Princípios e Técnicas. Editorial Universidad Federal de Vicosa. Vicosa, Minas Gerais, Brasil, 272 p.

Zobel, B y Talbert, J. 1984. Applied Forest Tree Improvement. John Wiley \& Sons. New York, USA, 510p.

Zobel, B. y Talbert, J. 1988. Técnicas de mejoramiento genético de arboles forestales. Ed. Limusa. México D.F, 545p 